\title{
Bayesian Analysis of the Discrete Two-Parameter Bathtub Hazard Distribution
}

\author{
Ammar M. Sarhan ${ }^{1}$ \\ ${ }^{1}$ Department of Mathematics and Statistics, Faculty of Science, Dalhousie University, Halifax, Canada
}

\section{Article Info}

Keywords: Data analysis, Maximum likelihood estimator, Probability models, Reliability measures 2010 AMS: 46N30, $90 B 25$

Received: 28 May 2019

Accepted: 18 September 2019

Available online: 26 December 2019

\begin{abstract}
A new discrete two-parameter bathtub hazard distribution is proposed by Sarhan [1]. This paper uses Bayes method to estimate the two unknown parameters and the reliability measures of this distribution. The joint posterior distribution of the model parameters cannot be obtained in a convenient form. Therefore, numerical techniques are needed. We apply four Bayesian numerical methods to get random draws from the joint posterior distribution to be used to estimate the model parameters and its reliability measures without deriving the actual joint posterior distribution. It is assumed here that the two model parameters are priori independent random variables with beta and gamma distributions. Two scenarios for the hyperparameters are applied to compare their contributions on the Bayesian inferences. Two real data sets are re-analyzed using the Bayesian techniques applied here. A simulation study is performed to investigate the properties of the methods applied.
\end{abstract}

\section{Introduction}

It is very common that the researchers in the fields of reliability analysis and life testing experiments use continues lifetime distributions to study the reliability of a system, see for example Gnedenko and Ushakov [2], Lawless [3], Sinha [4], and Kapur and Lamberson [5]. Sometimes, in practice, it is impossible to test the underlying system on a continuous scale. For example, the lifetime of the switch in the case of an on/off-switching unit, the number of cycles prior to failure of an equipment which operates in cycles, the lifetime (in days/weeks) of systems that are placed on the life test. In such situations, discrete lifetime distributions might be more appropriate.

Recently, Sarhan [1] proposed a discrete two-parameter distribution that displays bathtub shaped hazard function in addition to the increasing and decreasing shapes. The bathtub hazard shape property allows this model to fit a number of real datasets in reliability analysis. Sarhan [1] used DTPBT $(q, \beta)$ to denote this distribution, where $q$ and $\beta$ are the model parameters. He discussed some statistical properties of the $\operatorname{DTPBT}(q, \beta)$ and used some frequentest techniques such as quantile, least squares and maximum likelihood method to do inference on the two unknown parameters of the proposed model. Also, he used the model to analyze two real datasets and compared the proposed model with the discrete Weibull, (Nakagaw and Osaki [6]), discrete modified Weibull DMW (Nooghabi et al. [7]), discrete additive Weibull DAddW (Bebbington et al. [8]) and discrete reduced modified Weibull DRMW (Almalki and Nadarajah [9]). He concluded that the DTPBT model performed better fit than all of these above mentioned models.

The main goal of this current paper is to use Bayesian method to do inference on the DTPBT model. We use Bayesian method to estimate the model parameters (in point and interval). Also, we use Bayesian method to report on the reliability measures of the model. It is assumed here that the two parameters $q$ and $\beta$ are independent variables, where $q$ follows beta priori distribution with hyperparameters $\left(a_{1}, a_{2}\right)$ and $\beta$ follows a gamma priori distribution with hyperparameters $\left(b_{1}, b_{2}\right)$. Two scenarios for the hyperparameters $\left(a_{1}, a_{2}\right)$ and $\left(b_{1}, b_{2}\right)$ are proposed and a comparison between them is discussed. The posterior distribution of the vector of the two unknown parameters $\theta=(q, \beta)$, given the available data, cannot be derived in a convenient form. Therefore, numerical approaches for Bayesian analysis are needed. In this paper, we will apply: (1) the accept-reject (AR) technique, (2) the sampling importance resampling (SIR) method, and (3) two versions of Monte Carlo Markov chain (MCMC) algorithm to get random draws from the joint posterior distribution. Once the random draws from the joint posterior distribution are obtained, we can perform any Bayesian analysis we wish for the model parameters or any of the model reliability measures. 
One of the advantages of the Bayesian methods applied here is that they do not require the posterior distribution of $(q, \beta)$ to be in an explicit form.

The rest of the paper is organized as follows. In Section 2 we give a brief description of the DTPBT. In Section 3 , we present the joint posterior distribution of $\theta=(q, \beta)$ and discuss the Bayes analysis. Three main numerical methods to draw random samples from the joint posterior distribution are discussed in Section 4. In Section 5, two real data sets are analyzed using the proposed methods. A simulation study is carried out in section 6 . Finally, section 7 concludes the paper and discusses some future work.

\section{The $\operatorname{DTPBT}(q, \beta)$ distribution}

The discrete random variable $X$ is said to have a discrete two-parameter bathtub hazard distribution, with parameters $q$ and $\beta$, if its probability mass function takes the following form, Sarhan [1],

$$
p(x ; q, \beta)=\frac{1}{q}\left[q^{e^{(x-1)^{\beta}}}-q^{e^{\beta^{\beta}}}\right], x=1,2, \cdots, 0<q<1, \beta>0 .
$$

The survival function (sf) of $X$ is

$$
S(x ; q, \beta)=q^{e^{x^{\beta}}-1}, x=1,2, \cdots, 0<q<1, \beta>0 .
$$

The cumulative distribution function (cdf) of $X$ is

$$
F(x ; q, \beta)=1-q^{e^{x^{\beta}}-1}, x=1,2, \cdots, 0<q<1, \beta>0 .
$$

The hazard rate function (hrf) of $X$ is

$$
h(x ; q, \beta)=1-q^{e^{x^{\beta}}-e^{(x-1)^{\beta}}}, x=1,2, \cdots, 0<q<1, \beta>0 .
$$

Sarhan [1] shows that the hazard function can be increasing/decreasing or bathtub shaped based on the values of the model parameters. Example of the different shapes of the hazard rate function are provided in Figure 2.1 when $(q, \beta)$ is $(0.9,0.2)$ increasing, $(0.9,0.3)$ bathtub and $(0.9,0.9)$ decreasing. The variety of the shape of hazard function, specifically the bathtub shaped, allows the DTPBT distribution to fit various number of discrete real datasets.
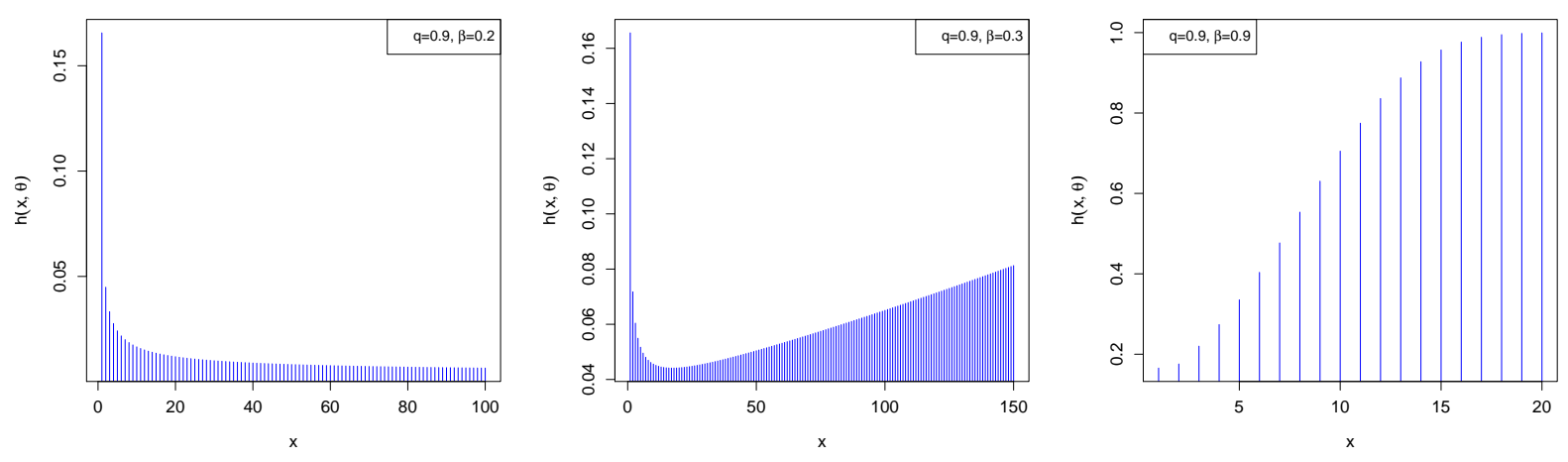

Figure 2.1: The hazard rate function of the DTPBT distribution when $(q, \beta)$ is [from the left to right] $(0.9,0.2),(0.9,0.3)$ and $(0.9,0.9)$.

\section{Bayes analysis}

Let $X_{1}, X_{2}, \cdots, X_{n}$ be a simple random sample from $\operatorname{DTPBT}(q, \beta)$. The likelihood function of this sample is

$$
L(\underline{x} ; q, \beta)=\frac{1}{q^{n}} \prod_{i=1}^{n}\left[q^{e^{\left(x_{i}-1\right)^{\beta}}}-q^{e^{e_{i}}}\right],
$$

where $\underline{x}$ represents the observations of the simple random sample. Let us assume that $q$ and $\beta$ be independent random variables with priori $\operatorname{beta}\left(a_{1}, a_{2}\right)$ and gamma $\left(b_{1}, b_{2}\right)$ distributions, respectively. That is, the joint prior density of $(q, \beta)$, up to a constant, is

$$
g(q, \beta) \propto q^{a_{1}-1}(1-q)^{a_{2}-1} \beta^{b_{1}-1} e^{-b_{2} \beta}, 0<q<1 ; \beta>0 .
$$

The hyperparameters $a_{j}, b_{j}>0, j=1,2$, will be subjectively selected based on the priori information on $q$ and $\beta$ when it is available. Applying Bayes' theorem using the likelihood function (3.1) and the joint prior density function (3.2), we get the joint posterior density function of $(q, \beta)$, given the data, up to a constant, as

$$
g(q, \beta \mid \underline{x}) \propto q^{-n+a_{1}-1}(1-q)^{a_{2}-1} \beta^{b_{1}-1} e^{-b_{2} \beta} \prod_{i=1}^{n}\left[q^{e^{\left(x_{i}-1\right) \beta}}-q^{e^{x_{i}^{\beta}}}\right], 0<q<1 ; \beta>0 .
$$

Under the squared error loss, which is adopted here, 
1. The Bayes estimates of $q$ and $\beta$ are the posterior means given by

$$
\hat{q}=\int_{0}^{1} q g_{q}(q \mid \underline{x}) d q \text { and } \hat{\beta}=\int_{0}^{\infty} \beta g_{\beta}(\beta \mid \underline{x}) d \beta,
$$

where $g_{q}(q \mid \underline{x})$ and $g_{\beta}(\beta \mid \underline{x})$ are the marginal posterior density functions of $q$ and $\beta$, respectively.

2. The Bayes estimate of a real-valued function of the $\vartheta=(q, \beta)$, say $w(q, \beta)$, is the posterior mean of $w(q, \beta)$, that is

$$
\hat{w}(q, \beta)=\int_{0}^{\infty} \int_{0}^{1} w(q, \beta) g(q, \beta \mid \underline{x}) d q d \beta .
$$

The joint posterior distribution (3.3) is in an inconvenient form as well as the normalized constant can not be derived theoretically. Therefore, the marginal posterior densities $g_{q}(q \mid \underline{x})$ and $g_{\beta}(\beta \mid \underline{x})$ cannot be derived explicitly, and so the integrals in (3.4) and (3.5) cannot be calculated analytically. Accordingly, we will use numerical approaches to perform Bayes analysis for the model parameters and its reliability measures. For the numerical techniques applied in this paper, it is more convenient to reparametrize the two parameters $q \in(0,1)$ and $\beta>0$ to be both real-valued by using the expressions $\theta_{1}=\log \left(\frac{q}{1-q}\right)$ and $\theta_{2}=\log (\beta)$. The joint posterior density function of $\theta=\left(\theta_{1}, \theta_{2}\right)$, given data, is

$$
g_{\theta}\left(\theta_{1}, \theta_{2} \mid \underline{x}\right)=g\left(\frac{1}{1+e^{-\theta_{1}}}, e^{\theta_{2}} \mid \underline{x}\right) \frac{e^{\theta_{2}-\theta_{1}}}{\left(1+e^{-\theta_{1}}\right)^{2}},-\infty<\theta_{1}, \theta_{2}<\infty .
$$

Also, for the numerical approaches which will be applied here, it is helpful to use the natural logarithm of the joint posterior density of $\theta$, which is given by

$$
\log g_{\theta}\left(\theta_{1}, \theta_{2} \mid \underline{x}\right)=\log g\left(\frac{1}{1+e^{-\theta_{1}}}, e^{\theta_{2}} \mid \underline{x}\right)+\theta_{2}-\theta_{1}-2 \log \left(1+e^{-\theta_{1}}\right) .
$$

\section{Bayesian numerical techniques}

In this section we discuss how to use four Bayesian numerical approaches that will help us to overcome the main difficulties in Bayes analysis. These techniques allow us to get random draws from the joint posterior distribution without deriving its explicit form. These techniques require only to write the posterior density function up to a constant as given in (3.3). For simplicity, in the rest of the paper, we use $\vartheta$ to denote the vector of the two unknown parameters, namely $\vartheta=(q, \beta)$.

Accept-Reject method (AR): The AR method is a general technique that can be used to generate independent random draws from probability distributions. It is one of the most useful techniques for simulating random draws from a variety of probability distributions. For more information on the AR method, we refer to Givens and Hoeting [10], Monahan [11], and Robert and Casella [12].

The main goal of using the AR method is to generate independent random draws from the posterior probability density (3.3). The basic idea in the AR method is to find a proposal, say $p(\vartheta)$, that satisfies: (1) easy to simulate from, (2) mimics the posterior distribution, and (3) there exits a positive constant $M$ such that $\frac{g(\vartheta \mid \text { data })}{p(\vartheta)} \leq M$ for all $\vartheta$. The following steps summarizes the AR algorithm:

1. Specify the size of desired random draws $m$.

2. Simulate $\vartheta$ from the proposal $p(\vartheta)$.

3. Calculate the ratio $R=\frac{g(\vartheta \mid \text { data })}{M p(\vartheta)}$.

4. Generate a random value $U$ from uniform distribution on the unit interval.

5. If $U \leq R$ accept $\vartheta$ as a random draw from $g(\vartheta \mid$ data); otherwise reject it.

6. Repeat steps 2-5 until $m$ draws are accepted.

We will use the bivariate $t$ distribution with a small degrees of freedom as a proposal since smaller degrees of freedom provide a heavy tailed proposal and therefore it would be more likely to find the upper bound constant $M$.

The location and variance-covariance matrix of the proposal distribution can be found as those we obtain by using normal approximation to the logarithm of the joint posterior distribution of the real-valued vector $\theta$, given in (3.6). Let $\kappa(\theta)=\log g_{\theta}(\theta \mid$ data $)$ and $\tilde{\theta}$ be its mode. The second-order Taylor series expansion of $\kappa(\theta)$ about $\tilde{\theta}$ is

$$
\kappa(\theta) \approx \kappa(\tilde{\theta})+\frac{1}{2}(\theta-\tilde{\theta})^{T} \kappa^{\prime \prime}(\tilde{\theta})(\theta-\tilde{\theta})
$$

where $\kappa^{\prime \prime}(\tilde{\theta})$ is the Hessian of $\kappa(\theta)$ evaluated at $\tilde{\theta}$. The above expansion of $\kappa(\theta)$ can be approximated by multivariate normal distribution with mean $\mu=\tilde{\theta}$ and variance-covariance matrix $\Sigma=\left(-\kappa^{\prime \prime}(\tilde{\theta})\right)^{-1}$. To apply this normal approximation, we need the mode $\tilde{\theta}$, that can be estimated by using Newton's method. Newton's method starts with a guess mode $\theta^{(0)}$, then estimates the mode at the $i$ th iteration by

$$
\theta^{(i)}=\theta^{(i-1)}-\left(\kappa^{\prime \prime}\left(\theta^{(i-1)}\right)\right)^{-1} \kappa^{\prime}\left(\theta^{(i-1)}\right)
$$

where $\kappa^{\prime}\left(\theta^{(i-1)}\right)$ and $\kappa^{\prime \prime}\left(\theta^{(i-1)}\right)$ are the gradient and Hessian of $\kappa(\theta)$ at the iteration $i-1$. These iterations are repeated until convergence. The constant $M$ can be found by maximizing $D(\theta)=\kappa(\theta)-\log p(\theta)$ with respect to $\theta$. Again, Newton's method can be used to obtain $\log M$ as the mode of $D(\theta)$, then calculate $M$. 
Sampling Importance Resampling Method (SIR): Sampling importance resampling (SIR) method is an alternative general method for simulating independent random draws from a general posterior distribution. As in the AR method, SIR requires a proposal that mimics the target posterior distribution but it does not require the bound constant $M$. We will use the same proposal as in the AR method. The SIR method can be implemented by applying the following algorithm:

1. Simulated $m$ draws from the proposal density $p(\vartheta)$, say $\vartheta^{(1)}, \cdots, \vartheta^{(m)}$

2. Compute the weights for the draws $w_{j}=\frac{g\left(\vartheta^{(i)} \mid \text { data }\right)}{p\left(\vartheta^{(j)}\right)}, i=1, \cdots, m$

3. Convert the weights to probabilities $w_{i}^{*}=\frac{w_{i}}{\sum_{j=1}^{M} w_{j}}, i=1, \cdots, m$

4. Simulate $m$ random draws with replacement from the discrete distribution $\left\{\vartheta^{(i)}, w_{i}^{*}\right\}_{i=1}^{m}$, say $\vartheta^{* 1}, \cdots, \vartheta^{* m}$.

Then the set of random draws $\left\{\vartheta^{* 1}, \cdots, \vartheta^{* m}\right\}$ is approximately distributed according to the actual posterior distribution $g(\vartheta \mid$ data).

Markov Chain Monte Carlo method (MCMC): Markov Chain Monte Carlo (MCMC) methods have been extensively used to become one of the most useful computational tools in the modern Bayesian data analysis. MCMC is very general and flexible technique to simulate a sequence of nonindependent draws from a probability distribution. MCMC method begins with an initial value $\vartheta^{(0)}$ and a mechanism for drawing the $\mathrm{i}$-th value in the sequence $\vartheta^{(i)}$ given the $(i-1)$ st value $\vartheta^{(i-1)}$. This mechanism consists of: (1) a proposal probability distribution $p\left(\vartheta^{*} \mid \vartheta\right)$ that produces a candidate value $\vartheta^{*}$ given a current $\vartheta$, and (2) calculate an acceptance probability $P$ of accepting the candidate $\vartheta^{*}$ as the next value in the sequence. A general reference for the MCMC is Gelman et al. [13].

The following steps can be followed to implement the Metropolis-Hastings algorithm:

1. Specify the sequence size $m>1$.

2. Choose an initial value $\vartheta^{(0)}=\left(\vartheta_{1}^{(0)}, \vartheta_{2}^{(0)}\right)^{\prime}$.

3. For $i=1, \cdots, m$ repeat the following steps

i. Set $\vartheta^{(i)}=\vartheta^{(i-1)}$

ii. Generate a candidate value $\vartheta^{*}$ from a proposal distribution $p\left(\vartheta^{*} \mid \vartheta^{(i)}\right)$.

iii. Calculate the ratio $r_{i}=\frac{g\left(\vartheta^{*} \mid \text { data }\right) / p\left(\vartheta^{*} \mid \vartheta^{(i)}\right)}{g\left(\theta^{(i)} \mid \text { data }\right) / p\left(\vartheta \mid \vartheta^{*}\right)}$.

$i v$. Set $\vartheta^{(i)}=\vartheta^{*}$ with probability $P_{i}=\min \left\{1, r_{i}\right\}$; otherwise keep $\vartheta^{(i)}$ as is.

Metropolis-Hastings algorithm (MHA) uses different proposals. However, we will use two different chains: (1) independence chain (IND), in which the acceptance ratio is $r_{i}=\frac{g\left(\vartheta^{*} \mid \text { data }\right) p\left(\vartheta^{(i)}\right)}{g\left(\vartheta^{(i)} \mid \text { data }\right) p\left(\vartheta^{*}\right)}$, and (2) random walk chain (RWC), in which the acceptance ratio is $r_{i}=\frac{g\left(\vartheta^{*} \mid \text { data }\right)}{g\left(\vartheta^{(i)} \mid \text { data) }\right.}$. The MHA generates random draws from the target distribution regardless of the proposal $p\left(\vartheta^{*} \mid \vartheta\right)$. However, the choice of the proposal is important since a poor choice considerably delays the convergence towards the target distribution. We will use the same proposal as that we use in the AR.

\section{Data analysis}

We use the four previously discussed Bayesian numerical methods to analyze the same two real datasets that were analyzed by Sarhan [1].

\subsection{Electronic devices data:}

In this dataset, 18 electronic devices were put on a life test and their lifetimes (in days) were observed as 5, 11, 21, 31, 46, 75, 98, 122, $145,165,196,224,245,293,321,330,350,420$. Wang [14] showed that this data have a bathtub hazard shape and used the additive Burr XII distribution to analyze it. Sarhan [1] applied different frequentest methods to re-analyze this dataset using the DTPBT distribution. In this section, we apply Bayes methods discussed here to analyze this dataset using the DTPBT distribution. Also, we estimate the model reliability measures.

Figure 5.1 [left panel] displays the contour plots for the log-posterior density function of $(q, \beta)$, which shows that the posterior distribution is right skewed and therefore normal approximation to the log-posterior distribution is not appropriate. This is one of the reasons to transform the two parameters $q$ from $(0,1)$ interval to the real valued interval using $\theta_{1}=\log \left(\frac{q}{1-q}\right)$ and $\beta$ from $(0, \infty)$ domain to real valued domain using $\theta_{2}=\log \beta$. Using this transformation, the $\log$-posterior density function becomes more symmetric as shown in Figure 5.1 [right panel] and therefore normal approximation for $\log g_{\theta}(\theta \mid$ data $)$ is appropriate. This will allow us to use the mode and variance covariance matrix for the normal approximation as the location and scale parameters for a symmetric proposal distribution. In this study, we use bivariate $\mathrm{t}$ distribution with 4 degrees of freedom as a proposal in all four Bayesian numerical methods.

We used beta and gamma prior distributions for the two unknown parameters $q$ and $\beta$, respectively, under two scenarios: (1) Poor/weak prior information, in which we assume that all hyperparameters are equal and equal to 0.001 , this indicates that prior mean of $q$ is 0.5 and standard deviation of 0.4995 , while $\beta$ has a prior mean of 1 and variance of 1000; (2) Good prior information: we use the posterior mean and posterior variance, that are obtained by using scenario 1 , as prior mean and variance for each parameter then get a new set of hyperparameters $a_{1}=168.594, a_{2}=1.419, b_{1}=141.829$ and $b_{2}=472.326$. As a quick diagnostic test for the effect of the prior information, we provided the contour plots for the log-likelihood function and the log-posterior density function of $(q, \beta)$ using each scenario along with the contour plots for the log-prior density function of $(q, \beta)$ using each scenario as displayed in Figure 5.2. Despite of scenario 1 provides a very skewed prior distribution, it slightly improves the skewness of the likelihood function. Scenario 2 is more symmetric and less distributed which leads to a less skewness in the joint posterior distribution of $(q, \beta)$ with more precision in the sense of having narrower contour.

Using scenario 1, the acceptance rates for the accept-reject (AR), random walk (RW) and independent chain (IND) Markov Chain Monte Carlo are $10.81 \%, 46.67 \%$ and $67.46 \%$, respectively. The AR methods has the lowest acceptance rate, then the RW and the INC has the 

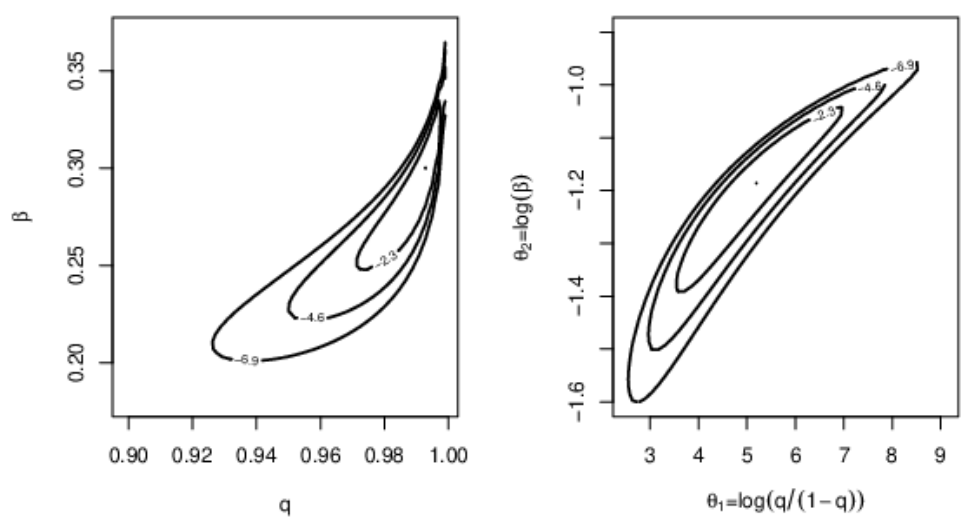

Figure 5.1: The contour plots for the log-posterior of $(q, \beta)$ [left panel] and for $\left(\log \left(\frac{q}{1-q}\right), \log \beta\right)$ [right panel], using non informative priors (scenario 1) of the hyperparameters.
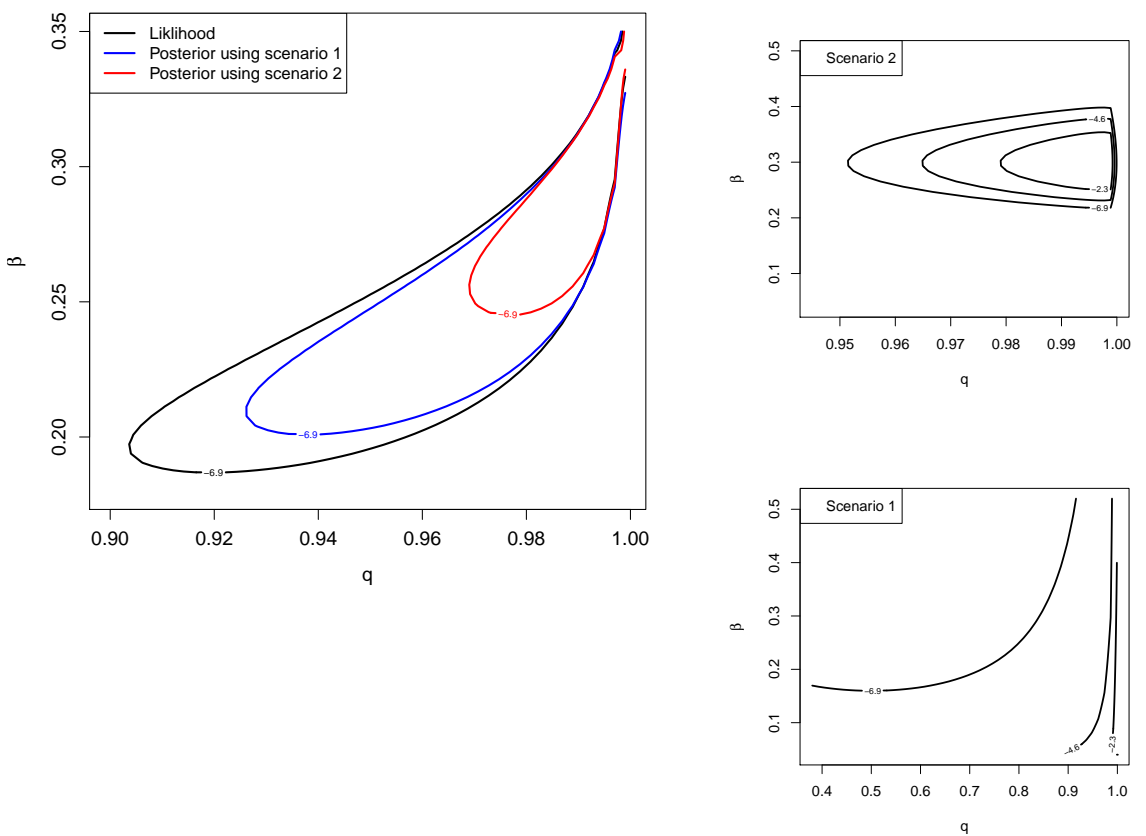

Figure 5.2: The contour plots [left panel] for the log-likelihood and for the log-posterior functions of $(q, \beta)$ using non informative priors (scenario 1 ) and informative priors (scenario 2); and the contour plots for the log-prior density using scenario 1 [bottom-right] and scenario 2 [top-right].

largest acceptance rate. The accept reject method is the slowest method comparing to the other three techniques applied here. The fastest one is the SIR method. The posterior mean, mode and 95\% credible interval for the two parameters $q$ and $\beta$ using the four techniques are displayed in Table 1 . The four techniques provide very similar results. However the INC provides more precise credible interval but the SIR is more faster and provides independent draws. Therefore, we would recommend SIR algorithm, for the underling distribution, over all other techniques discussed here.

Using scenario 2, the acceptance rates for AR, RW and IND algorithms are respectively $52.3 \%, 50.8 \%$ and $78.12 \%$, which are generally higher than those for scenario 1 . The acceptance rate corresponding to AR is increased by more than $40 \%$ using scenario 2 . Furthermore, as expected, scenario 2 provides better Bayesian inferences for $q$ and $\beta$ than scenario 1 in the sense of providing narrower credible intervals. However, all Bayesian point estimates are very similar.

The four numerical techniques are performed using prior scenario 1 to get 10,000 draws from the posterior distribution of the transformed parameters $\theta=\left(\theta_{1}, \theta_{2}\right)$. Figure 5.3 shows the posterior contours along with the draws obtained using the four methods. Using the inverse transformations of $\theta_{1}$ and $\theta_{2}, q=\left(1+e^{-\theta_{1}}\right)^{-1}$ and $\beta=e^{\theta_{2}}$, we get random draws from the marginal posterior distributions of $q$ and $\beta$, given data, respectively. Figure 5.4 shows the marginal posterior densities of $q$ and $\beta$ using the four techniques. To see how good the draws that are obtained from the MCMC, RW and IND chains, we provide some diagnostic tests; the trace plots and autocorrelation plots for $\theta_{1}$ and $\theta_{2}$ using the whole chains and the last $70 \%$ of the chains. From these plots, we can see that there is a good mix of the draws and the Lag 
declines rapidly in addition to the high acceptance rates of both two chains. The trace plots show that the early $27 \%$ of the chain were not plausible values from the posterior distribution. That is why we discarded the early $30 \%$ of the chain and used the last $70 \%$ as draws from the actual posterior distribution. Also, the ACF plots show high correlation in the chain over the early $30 \%$ (burn-in period), then declines rapidly in the last $70 \%$.

\begin{tabular}{llccccc}
\multirow{2}{*}{ Parameter } & Method & Mean & Median & 2.5th & 97.5 th & Width \\
\hline \multirow{4}{*}{$q$} & & & & Scenario 1 \\
& SIR & 0.991305 & 0.993312 & 0.972841 & 0.998798 & 0.025956 \\
& AR & 0.991379 & 0.993427 & 0.972511 & 0.998784 & 0.026273 \\
\multirow{4}{*}{$\beta$} & RW & 0.991258 & 0.993529 & 0.970904 & 0.998572 & 0.027668 \\
& IND & 0.991850 & 0.993608 & 0.974821 & 0.998804 & 0.023983 \\
\cline { 2 - 7 } & SIR & 0.298777 & 0.299823 & 0.246841 & 0.345158 & 0.098318 \\
& AR & 0.299521 & 0.300323 & 0.247899 & 0.346341 & 0.098442 \\
& RW & 0.299066 & 0.300799 & 0.245420 & 0.342906 & 0.097486 \\
& IND & 0.300765 & 0.300979 & 0.252553 & 0.346116 & 0.093563 \\
\hline \multirow{4}{*}{$\beta$} & & & & Scenario 2 & & \\
\cline { 2 - 7 }$\beta$ & SIR & 0.992372 & 0.992988 & 0.983703 & 0.997554 & 0.013852 \\
& AR & 0.992372 & 0.993072 & 0.983525 & 0.997504 & 0.013978 \\
& RW & 0.992154 & 0.993034 & 0.981538 & 0.998019 & 0.025692 \\
& IND & 0.992357 & 0.993032 & 0.983500 & 0.997490 & 0.013990 \\
\cline { 2 - 7 } & SIR & 0.298861 & 0.298871 & 0.269706 & 0.328245 & 0.058540 \\
& AR & 0.299227 & 0.299151 & 0.269963 & 0.328406 & 0.058443 \\
& RW & 0.285673 & 0.298209 & 0.001915 & 0.330015 & 0.082522 \\
& IND & 0.298920 & 0.299080 & 0.269414 & 0.328720 & 0.059306 \\
\hline
\end{tabular}

Table 1: The posterior mean, median, 2.5th, 97.5 th percentiles (the bounds of the $95 \%$ credible interval) and the credible intervals' widths of $q$ and $\beta$, using scenarios 1 and 2.

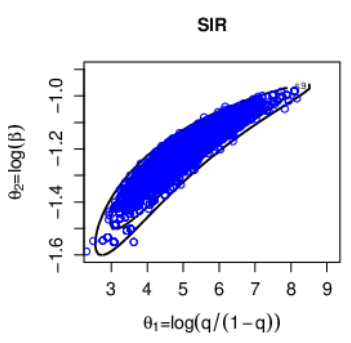

SIR

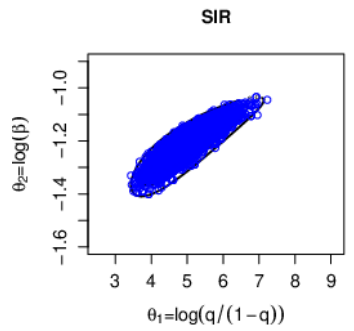

AR

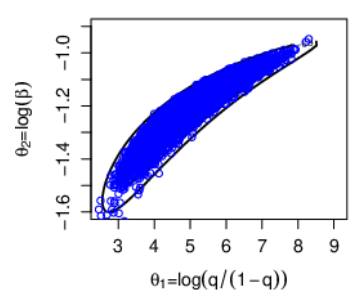

AR

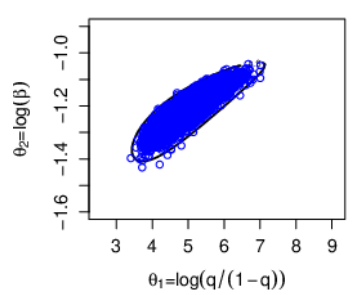

MCMC - RW

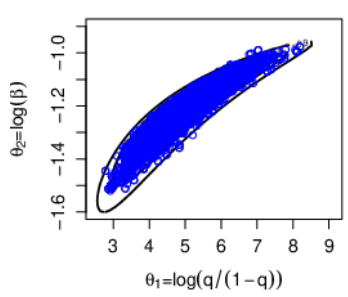

MCMC-RW

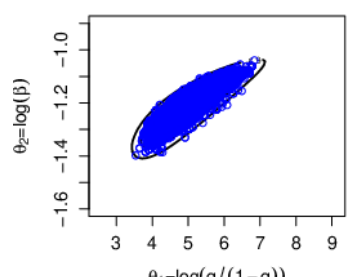

MCMC - IND
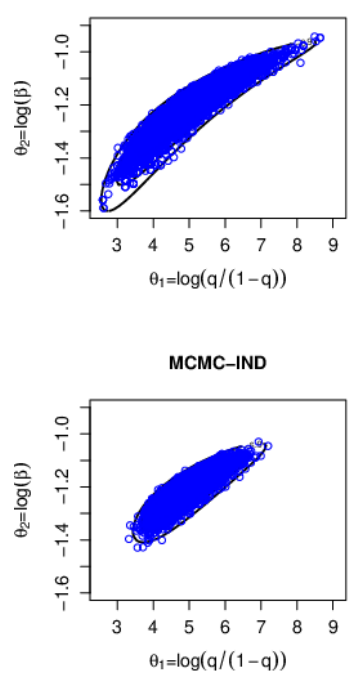

Figure 5.3: The contour plots for the $\log$-posterior of $\left(\log \left(\frac{q}{1-q}\right), \log \beta\right)$ along with the draws obtained from the joint posterior distribution using SIR, AR, MCMC-RW and MCMC-IND methods using scenario 1 [top row] and scenario 2 [bottm row].

We can use the draws that were simulated from the joint posterior distribution of $(q, \beta)$, by following the adopted technique, to get random draws from the posterior distribution of any reliability measure we wish without deriving the actual posterior distribution of that measure. As examples, we calculate the basic Bayes analysis for the probability mass function and the reliability function of the model at a given value $x_{0}$, e.g., the sample mean, using each adopted technique in this paper using the two scenarios of the hyperparameters. Table 2 shows the posterior mean, median, the limits of a $95 \%$ credible interval of each measure.

Figure 5.6 displays the Bayes estimate of the posterior probability density function of $P\left(X=x_{0}\right)$, when $x_{0}=172$ 'the sample mean', using the four techniques applied here and the two scenarios of the hyperparameters. As expected, the posterior distribution of $p(172)$ using prior scenario 1 has more spread than that obtained using prior scenario 2 .

Both the two prior scenarios provide similar results for the probability mass and survival functions that shows the robustness of the Bayesian analysis. 

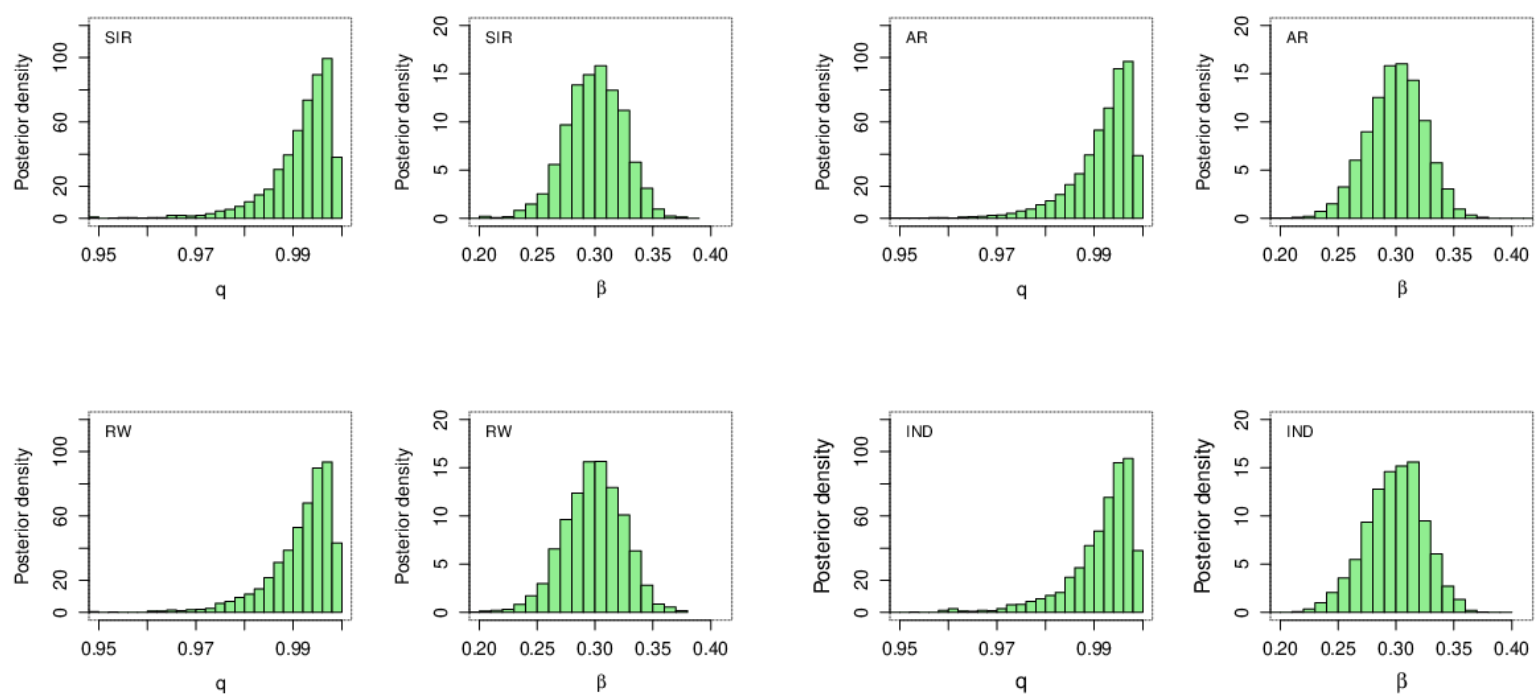

Figure 5.4: The approximated marginal posterior density functions of $q$ and $\beta$ using SIR (top left), AR (top right), RW (bottom left) and IND (bottom right) methods, using scenario 1 of the hyperparameters.
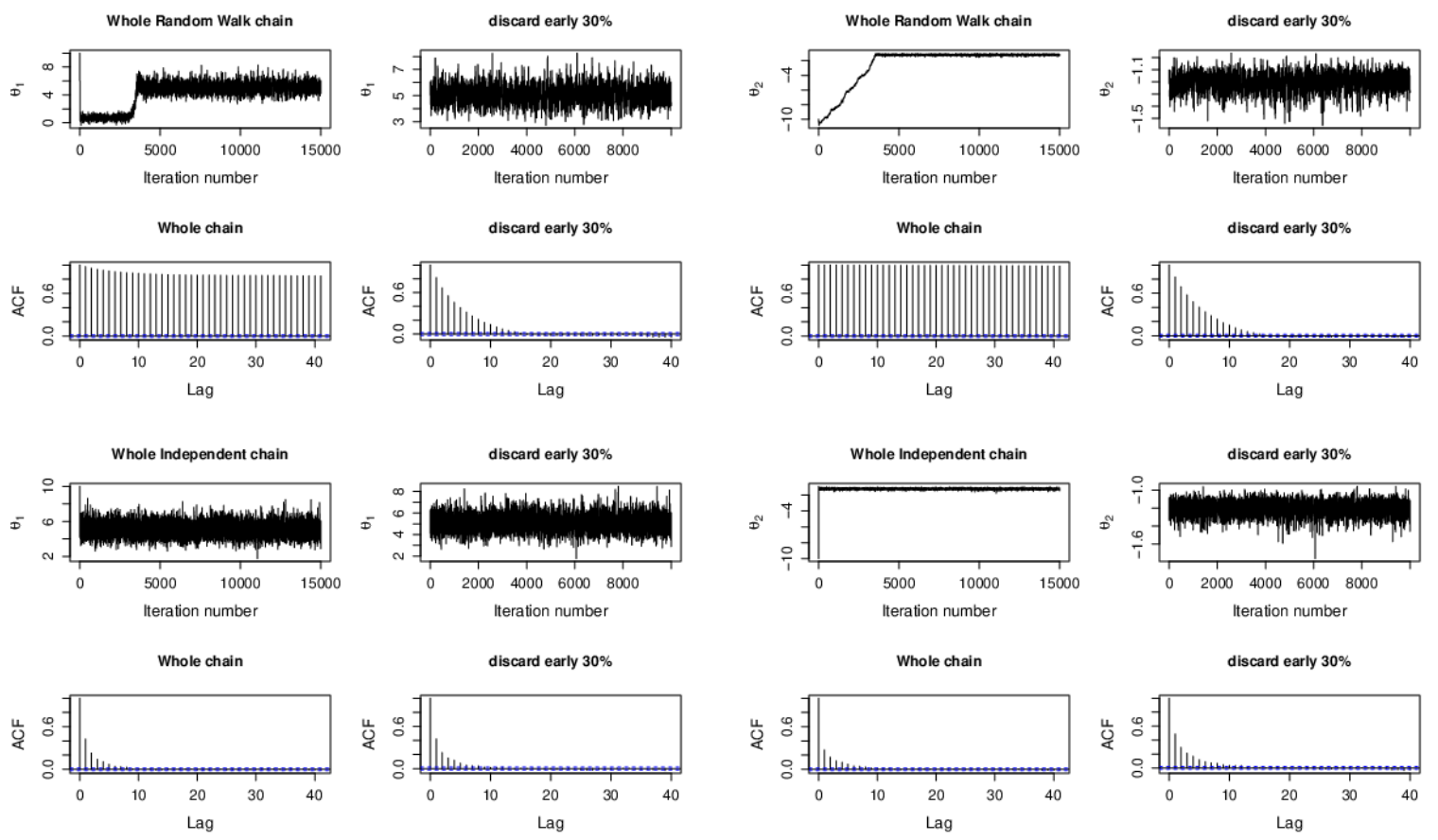

Figure 5.5: The trace and autocorrelation plots for the transformed parameters using RW [top two rows] and IND [bottom two rows]

\subsection{Aarset data:}

Aarset data [15] consist of the failure times (in weeks) of 50 devices put on a life test. The TTT-Transform plot for this dataset shows that it has a bathtub-shape. Nooghabi et al. [7] used the DMW distribution, Xie and Lai [16] used DAddW, and Almalki and Nadarajah [9] used DRMW to analyze this data set and reported that all of these distributions fit the data well. Sarhan [1] used the DTPBT distribution to analyze this dataset and compared it with those models mentioned above and reported that the DTPBT was better.

In the first dataset, all Bayesian point estimates were very similar using both scenarios of the hyperparameters. Therefore, in this dataset, we implement all the Bayesian techniques adopted here using only scenario 1 of the hyperparameters. Table 3 presents the posterior mean, median and the limits of the $95 \%$ credible interval along with its width of the model parameters and the probability mass function and survival function of a random selected device evaluated at 46 weeks (the sample mean). As in the first dataset, all the Bayesian methods, we applied here, give similar results. 


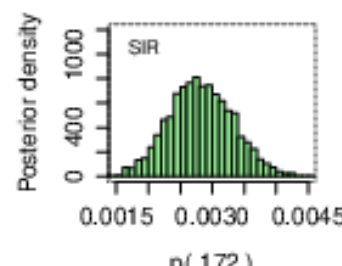

$p(172)$
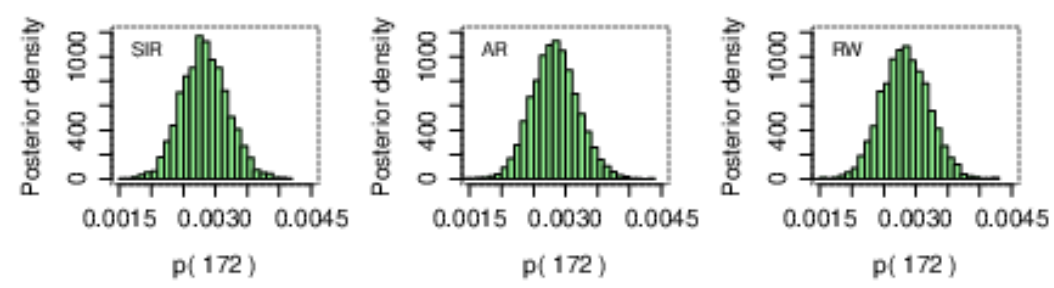

Figure 5.6: The approximated posterior density function of $p(172)=P(X=172)$ using the four techniques SIR, AR, RW, IND by applying scenario 1 [top row] and scenario 2 [bottom row].
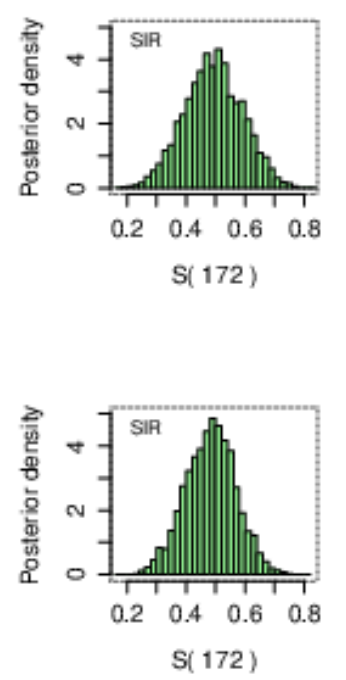
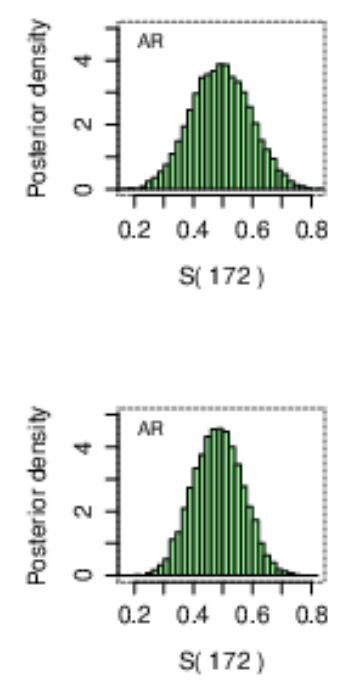
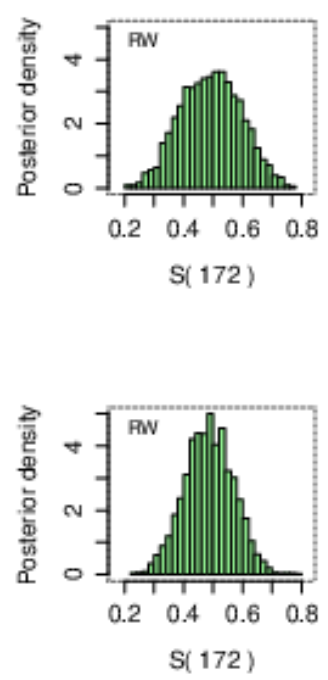
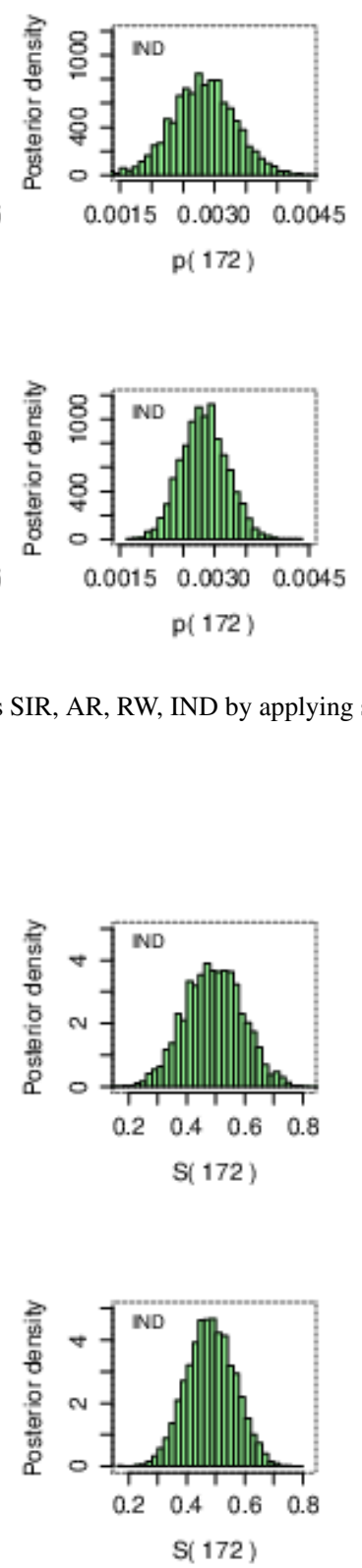

Figure 5.7: The approximated posterior density function of $S(172)$ using the four techniques SIR, AR, RW, IND [from left to right] using scenario 1 [top row] and scenario 2 [bottom row].

\section{Simulation results}

In order to compare the performance of the four numerical methods applied here, a simulation study is implemented according to the following scheme:

1. Specify the actual value of the vector of model parameters $\vartheta=(q, \beta)$

2. Specify the sample size $n$

3. Generate a random sample with size $n$ from the $\operatorname{DTPBT}(q, \beta)$

4. For the random sample obtained in step 3,

(a) generate $m$ random draws from the joint posterior distribution by applying each of the four simulation techniques described in this article (AR, SIR, RW and IND)

(b) use the random draws obtained in (a), using every technique, to calculate the Bayes estimate and 95\% Bayesian probability interval 'BPI' for $q$ and $\beta$

(c) for every parameter, calculate: (i) the bias 'exact - estimate' and (ii) an indicator $C=1$ if the BPI captures the actual value and 0 , otherwise.

5. Repeat Steps 3 and 4, $N$ times, then calculate the mean squared errors 'MSE' and the coverage probability 'CP' for every parameter, 


\begin{tabular}{|c|c|c|c|c|c|c|}
\hline Measure & Method & Mean & Median & 2.5th & 97.5th & Width \\
\hline \multirow{3}{*}{$p(172)$} & & \multicolumn{5}{|c|}{ Scenario 1} \\
\hline & SIR & 0.00282 & 0.00281 & 0.00187 & 0th.00380 & 0.00193 \\
\hline & $\mathrm{AR}$ & 0.00282 & 0.00282 & 0.00190 & 0.00379 & 0.00190 \\
\hline \multirow{6}{*}{$S(172)$} & RW & 0.00280 & 0.00280 & 0.00186 & 0.00379 & 0.00193 \\
\hline & IND & 0.00280 & 0.00281 & 0.00183 & 0.00381 & 0.00198 \\
\hline & SIR & 0.49126 & 0.49213 & 0.29913 & 0.68191 & 0.38278 \\
\hline & AR & 0.49275 & 0.49159 & 0.30592 & 0.68669 & 0.38077 \\
\hline & RW & 0.49433 & 0.49505 & 0.30318 & 0.68154 & 0.37836 \\
\hline & IND & 0.49251 & 0.49329 & 0.30049 & 0.68598 & 0.38549 \\
\hline \multirow[b]{2}{*}{$p(172)$} & & \multicolumn{5}{|c|}{ Scenario 2} \\
\hline & SIR & 0.00282 & 0.00281 & 0.00215 & 0.00357 & 0.00142 \\
\hline \multirow{7}{*}{$S(172)$} & AR & 0.00283 & 0.00282 & 0.00214 & 0.00357 & 0.00143 \\
\hline & RW & 0.00283 & 0.00282 & 0.00216 & 0.00359 & 0.00143 \\
\hline & IND & 0.00283 & 0.00284 & 0.00212 & 0.00357 & 0.00145 \\
\hline & SIR & 0.48475 & 0.48378 & 0.32239 & 0.65225 & 0.32986 \\
\hline & AR & 0.48338 & 0.48283 & 0.32064 & 0.64691 & 0.32627 \\
\hline & RW & 0.48597 & 0.48429 & 0.32773 & 0.65650 & 0.32877 \\
\hline & IND & 0.48487 & 0.48356 & 0.32330 & 0.64864 & 0.32534 \\
\hline
\end{tabular}

Table 2: Posterior mean, median, 2.5th, 97.5th percentiles (the bounds of the credible interval) and the credible intervals' widths of $p(172)$ and $S(172)$ using prior scenarios 1 and 2 .

\begin{tabular}{llccccc} 
Parameter & Method & Mean & Median & 2.5 th & 97.5th & Width \\
\hline$q$ & SIR & 0.97704 & 0.97872 & 0.95273 & 0.99130 & 0.03857 \\
& AR & 0.97703 & 0.97875 & 0.95344 & 0.99124 & 0.03780 \\
& RW & 0.97647 & 0.97809 & 0.95143 & 0.99112 & 0.03969 \\
$\beta$ & IND & 0.97684 & 0.97849 & 0.95263 & 0.99082 & 0.03819 \\
\cline { 2 - 6 }$\beta$ & SIR & 0.34020 & 0.34047 & 0.29433 & 0.38290 & 0.08857 \\
& AR & 0.34059 & 0.34093 & 0.29685 & 0.38268 & 0.08582 \\
& RW & 0.33885 & 0.33872 & 0.29409 & 0.38228 & 0.08819 \\
$p(46)$ & IND & 0.33976 & 0.34042 & 0.29456 & 0.38087 & 0.08631 \\
\cline { 2 - 6 }$S(46)$ & SIR & 0.01012 & 0.01008 & 0.00759 & 0.01279 & 0.00520 \\
& AR & 0.01013 & 0.01011 & 0.00769 & 0.01275 & 0.00505 \\
& RW & 0.01004 & 0.00998 & 0.00750 & 0.01266 & 0.00516 \\
& IND & 0.01010 & 0.01009 & 0.00758 & 0.01269 & 0.00511 \\
\cline { 2 - 6 } & SIR & 0.43495 & 0.43291 & 0.32432 & 0.55333 & 0.22901 \\
& AR & 0.43374 & 0.43318 & 0.31956 & 0.55621 & 0.23665 \\
& RW & 0.43293 & 0.43273 & 0.31709 & 0.54725 & 0.23016 \\
& IND & 0.43276 & 0.43372 & 0.31980 & 0.54828 & 0.22847 \\
\hline
\end{tabular}

Table 3: The posterior mean, median, 2.5 th, 97.5 th percentiles and the credible intervals' widths of $q, \beta$ and $p(46)$ and $S(46)$ using prior scenarios 1 .

using each technique, according to

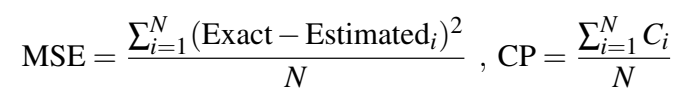

here Estimated ${ }_{i}$ and $C_{i}$ are the estimated value and the value of the indicator $C$ obtained in the $i$-th iteration.

We implemented the above algorithm using scenario 1 of hyperparameters with $N=10000, m=10000$, for different sample size $n=$ $25,50,75$, and 100 , and different sets of parameters' values $\vartheta=(0.9,0.2),(0.9,0.3)$ and $(0.9,0.9)$. The choice of the parameters' value was to reflect different shapes of the hazard function (decreasing, bathtub and increasing) of the DTPBT model. Table 4 summarizes the obtained results, from which we can conclude that: (1) the MSE decreases with the sample size for every parameter using all four numerical methods applied in this paper, (2) the MSE corresponding to each parameter is almost the same for each method, (3) the CP corresponding to each parameter using all four methods gets closer to the nominated confidence level when the samlpe size gets bigger.

\section{Conclusion}

Bayesian inferences of the two parameters of the discrete tow-parameter bathtub distribution and its reliability measures are discussed in this paper. The joint posterior distribution of the two parameters has no explicit form. To overcome this problem, four numerical Bayesian computation techniques (accept-reject, sampling-importance resampling and two versions of Markov chain Monte Carlo) are adopted.

The two parameters are assumed to be independent and beta and gamma priori distributed with known hyperparameters. Two scenarios of the hyperparameters are discussed and we showed that the Bayes analysis is robust for the choice of the hyperparameters. We re-analyzed two real datasets using the four Bayesian numerical techniques applied here. Finally, we performed a simulation study to investigate the properties of the four Bayesian methods. 
As a future work, the methods discussed in this paper can be used under different types of data sets with different testing scenarios such as progressively censored data, competing risks data and/or masked data.

\begin{tabular}{|c|c|c|c|c|c|c|c|c|c|}
\hline Parameter & \multicolumn{9}{|c|}{ Case 1: $(q, \beta)=(0.9,0.2)$} \\
\hline \multirow{8}{*}{$q$} & \multirow[t]{2}{*}{$n$} & \multicolumn{4}{|c|}{ MSE } & \multicolumn{4}{|c|}{$\mathrm{CP}$} \\
\hline & & AR & SIR & RW & IND & $\mathrm{AR}$ & SIR & RW & IND \\
\hline & 15 & 0.00196 & 0.00197 & 0.00198 & 0.00186 & 0.891 & 0.894 & 0.895 & 0.869 \\
\hline & 20 & 0.00145 & 0.00144 & 0.00145 & 0.00137 & 0.927 & 0.929 & 0.921 & 0.911 \\
\hline & 25 & 0.00108 & 0.00107 & 0.00108 & 0.00107 & 0.932 & 0.933 & 0.929 & 0.927 \\
\hline & 50 & 0.00057 & 0.00056 & 0.00056 & 0.00056 & 0.932 & 0.930 & 0.932 & 0.926 \\
\hline & 75 & 0.00040 & 0.00041 & 0.00041 & 0.00040 & 0.922 & 0.928 & 0.924 & 0.931 \\
\hline & 100 & 0.00031 & 0.00031 & 0.00031 & 0.00031 & 0.925 & 0.937 & 0.925 & 0.930 \\
\hline \multirow[t]{6}{*}{$\beta$} & 15 & 0.00125 & 0.00125 & 0.00125 & 0.00100 & 0.902 & 0.903 & 0.907 & 0.858 \\
\hline & 20 & 0.00078 & 0.00077 & 0.00077 & 0.00065 & 0.929 & 0.928 & 0.924 & 0.900 \\
\hline & 25 & 0.00049 & 0.00049 & 0.00049 & 0.00047 & 0.940 & 0.941 & 0.941 & 0.922 \\
\hline & 50 & 0.00025 & 0.00025 & 0.00025 & 0.00024 & 0.930 & 0.932 & 0.934 & 0.931 \\
\hline & 75 & 0.00017 & 0.00017 & 0.00017 & 0.00016 & 0.919 & 0.921 & 0.920 & 0.921 \\
\hline & 100 & 0.00012 & 0.00012 & 0.00012 & 0.00012 & 0.928 & 0.929 & 0.929 & 0.928 \\
\hline \multirow{7}{*}{$q$} & \multicolumn{9}{|c|}{ Case 2: $(q, \beta)=(0.9,0.3)$} \\
\hline & 15 & 0.00201 & 0.00201 & 0.00202 & 0.00193 & 0.894 & 0.908 & 0.901 & 0.869 \\
\hline & 20 & 0.00143 & 0.00142 & 0.00142 & 0.00138 & 0.911 & 0.913 & 0.917 & 0.894 \\
\hline & 25 & 0.00118 & 0.00117 & 0.00116 & 0.00115 & 0.921 & 0.922 & 0.916 & 0.909 \\
\hline & 50 & 0.00059 & 0.00059 & 0.00059 & 0.00059 & 0.930 & 0.932 & 0.930 & 0.932 \\
\hline & 75 & 0.00038 & 0.00038 & 0.00038 & 0.00038 & 0.938 & 0.941 & 0.937 & 0.938 \\
\hline & 100 & 0.00031 & 0.00031 & 0.00031 & 0.00031 & 0.923 & 0.927 & 0.930 & 0.924 \\
\hline \multirow[t]{6}{*}{$\beta$} & 15 & 0.00260 & 0.00261 & 0.00259 & 0.00204 & 0.930 & 0.925 & 0.922 & 0.874 \\
\hline & 20 & 0.00172 & 0.00172 & 0.00169 & 0.00153 & 0.917 & 0.918 & 0.917 & 0.898 \\
\hline & 25 & 0.00813 & 0.00814 & 0.00812 & 0.00742 & 0.914 & 0.912 & 0.916 & 0.905 \\
\hline & 50 & 0.00325 & 0.00325 & 0.00326 & 0.00325 & 0.942 & 0.939 & 0.935 & 0.931 \\
\hline & 75 & 0.00191 & 0.00190 & 0.00190 & 0.00189 & 0.941 & 0.944 & 0.941 & 0.944 \\
\hline & 100 & 0.00152 & 0.00151 & 0.00152 & 0.00152 & 0.933 & 0.929 & 0.933 & 0.920 \\
\hline \multirow{7}{*}{$q$} & \multicolumn{9}{|c|}{ Case 3: $(q, \beta)=(0.9,0.9)$} \\
\hline & 15 & 0.00253 & 0.00254 & 0.00255 & 0.00252 & 0.886 & 0.882 & 0.882 & 0.862 \\
\hline & 20 & 0.00164 & 0.00164 & 0.00165 & 0.00160 & 0.897 & 0.895 & 0.890 & 0.877 \\
\hline & 25 & 0.00128 & 0.00127 & 0.00127 & 0.00125 & 0.903 & 0.896 & 0.901 & 0.895 \\
\hline & 50 & 0.00057 & 0.00056 & 0.00057 & 0.00057 & 0.936 & 0.932 & 0.936 & 0.929 \\
\hline & 75 & 0.00041 & 0.00041 & 0.00041 & 0.00041 & 0.925 & 0.931 & 0.925 & 0.935 \\
\hline & 100 & 0.00029 & 0.00030 & 0.00029 & 0.00029 & 0.942 & 0.937 & 0.939 & 0.943 \\
\hline \multirow[t]{6}{*}{$\beta$} & 15 & 0.03212 & 0.03213 & 0.03208 & 0.03164 & 0.873 & 0.873 & 0.870 & 0.856 \\
\hline & 20 & 0.02818 & 0.02818 & 0.02801 & 0.02464 & 0.895 & 0.895 & 0.882 & 0.869 \\
\hline & 25 & 0.01787 & 0.01795 & 0.01757 & 0.01643 & 0.906 & 0.908 & 0.900 & 0.889 \\
\hline & 50 & 0.00622 & 0.00622 & 0.00621 & 0.00616 & 0.929 & 0.928 & 0.935 & 0.931 \\
\hline & 75 & 0.00415 & 0.00413 & 0.00413 & 0.00411 & 0.923 & 0.936 & 0.918 & 0.925 \\
\hline & 100 & 0.00277 & 0.00278 & 0.00276 & 0.00277 & 0.931 & 0.931 & 0.930 & 0.935 \\
\hline
\end{tabular}

Table 4: The MSE associated with the Bayes point estimate of the model parameters using each Bayesian simulation method and the coverage probability corresponding to the $95 \%$ credible intervals

\section{References}

[1] A. M. Sarhan, A two-parameter discrete distribution with a bathtub hazard shape, Commun. Stat. Appl. Methods, 24(1) (2017), $15-27$.

[2] B. Gnedenko, I. Ushakov, Probabilistic Reliability Engineering, John Wiley \& Sons, New York, 1995.

[3] J. F. Lawless, Statistical Models and Methods for Lifetime Data, John Wiley \& Sons, New York, 1982.

[4] S. K. Sinha, Reliability and Life testing, Wiley Eastern Ltd, New Delhi, 1986.

[5] K. C. Kapur KC, L. R. Lamberson, Reliability and in Engineering Design, John Wiley \& Sons, New York, 1976.

[6] T. Nakagawa, S. Osaki, The Discrete Weibull Distribution, IEEE Trans. Reliab., R-24(5) (1975), 300-301.

[7] M. S. Nooghabi, A. R. Roknabady G. H. Borzadaran, Discrete modified Weibull distribution, Metron, LXIX (2011), $207-222$.

[8] M. Bebbington, C-D Lai, M. Wellington, R. Zitikis, The discrete additive Weibull distribution: A bathtub-shaped hazard for discontinuous failure data, Reliab. Engrg. Syst. Safety, 106 (2012), 37-44.

[9] S. J. Almalki, S. Nadarajah, A new discrete modified Weibull distribution, IEEE Trans. Reliab., 63(1) (2014), 68-80.

[10] G. Givens, J. Hoeting, Computational Statistics, Hoboken. NJ: John Wiley and Sons, 2005.

[11] J. Monahan, Numerical Methods of Statistics, Cambridge: Cambridge University Press, 2001.

[12] C. Robert, G. Casella, Monte Carlo Statistical Methods, New York: Springer, 2014.

[13] A. Gelman, J. Carlin, H. Stern, D. Rubin, Bayesian Data Analysis, New York: Chapman and Hall, 2003.

[14] F. K. Wang, A new model with bathtub-shaped failure rate using an additive Burr XII distribution, Reliab. Engrg. Syst. Safety, 70 (2000), $305-312$.

[15] M. V. Aarset, How to identify a bathtub hazard rate, IEEE Trans. Reliab., R-36 (1987), 106-108.

[16] M. Xie, C. D. Lai, Reliability analysis using an additive Weibull model with bathtub-shaped failure rate function, Reliab. Engrg. Syst. Safety, 52 (1995), $87-93$. 